\title{
Nanomedicine in cancer therapy: promises and hurdles of polymeric nanoparticles
}

\author{
Carmen Paus ${ }^{1,2}$, Robbert van der Voort ${ }^{2}$, Alessandra Cambi ${ }^{1}{ }^{*}$ \\ ${ }^{1}$ Department of Cell Biology, Radboud Institute for Molecular Life Sciences, Radboud University Medical Center, 6525 GA, \\ Nijmegen, The Netherlands \\ ${ }^{2}$ Vision Medical Communications, 6525 JL, Nijmegen, The Netherlands
}

*Correspondence: Alessandra Cambi, Department of Cell Biology, Radboud Institute for Molecular Life Sciences, Radboud University Medical Center, Geert Grooteplein Zuid 26-28, 6525 GA, Nijmegen, The Netherlands. alessandra.cambi@ radboudumc.nl

Academic Editor: Yang Shi, RWTH Aachen University Clinic, Germany

Received: February 03, 2021 Accepted: March 28, 2021 Published: April 30, 2021

Cite this article: Paus C, van der Voort R, Cambi A. Nanomedicine in cancer therapy: promises and hurdles of polymeric nanoparticles. Explor Med. 2021;2:167-85. https://doi.org/10.37349/emed.2021.00040

\begin{abstract}
The limitations of current cancer treatments have stimulated the application of nanotechnology to develop more effective and safer cancer therapies. Remarkable progress has been made in the development of nanomedicine to overcome issues associated with conventional cancer treatment, including low drug solubility, insufficient targeting, and drug resistance. The modulation of nanoparticles allows the improvement of drug pharmacokinetics, leading to improved targeting and reduced side effects. In addition, nanoparticles can be conjugated to ligands that specifically target cancer cells. Furthermore, strategies that exploit tumor characteristics to locally trigger drug release have shown to increase targeted drug delivery. However, although some clinical successes have been achieved, most nanomedicines fail to reach the clinic. Factors that hinder clinical translation vary from the complexity of design, incomplete understanding of biological mechanisms, and high demands during the manufacturing process. Clinical translation might be improved by combining knowledge from different disciplines such as cell biology, chemistry, and tumor pathophysiology. An increased understanding on how nanoparticle modifications affect biological systems is pivotal to improve design, eventually aiding development of more effective nanomedicines. This review summarizes the key successes that have been made in nanomedicine, including improved drug delivery and release by polymeric nanoparticles as well as the introduction of strategies that overcome drug resistance. In addition, the application of nanomedicine in immunotherapy is discussed, and several remaining challenges addressed.
\end{abstract}

\section{Keywords}

Nanomedicine, cancer therapy, drug delivery, targeting, controlled drug release, clinical translation 


\section{Introduction}

Despite remarkable progress in our understanding of the biological background of cancer and the continuous improvement of cancer therapies, cancer remains one of the leading causes of death. Conventional therapies have improved patient survival but suffer from several limitations. For instance, anti-cancer drugs frequently fail because of their poor tumor selectivity and high toxicity in healthy tissue. Even when the treatment is initially effective, development of drug resistance is a common threat for long-term survival and cure $[1,2]$.

Nanomedicine is a novel and rapidly evolving field that might overcome many of the limitations of current anti-cancer drugs. Its nanosize, material and ability for surface modification provide unique features that can dramatically improve pharmacokinetics of incorporated therapeutic agents. Modifications can improve solubility, prolong circulation time, avoid clearance by the immune system or allow for controlled drug release. The nanosize allows drug accumulation in tumor tissue by passive targeting, which relies on the enhanced permeability and retention (EPR) effect. Active targeting on the other hand, comprises functionalized nanoparticles that specifically target molecules expressed on cancer cells, as this specific targeting has shown promise to increase the drug concentration at the site of action while limiting side effects.

The possibility for specific targeting also lends itself to individualized therapies. Tumor heterogeneity between patients is one of the main challenges in the development of effective drugs, and has prompted the emergence of personalized cancer medicine. Recent advances in nanomedicine have the potential to develop into effective and personalized anti-cancer therapies.

Over the past years, various nanoparticles have been developed for drug delivery to the tumor, including viruses, lipid based and polymeric nanoparticles [3]. This has led to a large variation in shape (e.g., spherical or rod-like), size, surface coating and material (e.g., lipid layer or polymer aggregate). These physicochemical properties can influence pharmacokinetic and biological parameters such as functionalization, toxicity, drug release, etc. (Table 1). Several modifications of nanoparticle physicochemical properties aimed at improving pharmacokinetics and targeting, or at reducing immunogenicity and toxicity have been reported (reviewed in $[4,5])$.

Table 1. Nanoparticle physicochemical properties influence pharmacokinetic and biological parameters

\begin{tabular}{llll}
\hline Shape & Size & Surface & Material \\
\hline Functionalization & Toxicity & Toxicity & Toxicity \\
Toxicity & Biodistribution & Biodistribution & Biodistribution \\
Stability & Opsonization & Stability & Opsonization \\
Biodistribution & Cellular uptake & Opsonization & Cellular uptake \\
Opsonization & Clearance & Tissue retention & Drug release \\
Cellular uptake & EPR effect & & Clearance \\
Tissue retention & Tissue retention & & Tissue retention \\
\hline
\end{tabular}

The possibility of modifying nanoparticle properties to circumvent limitations of current cancer drugs stimulated rapid progress to clinical use. Several therapeutic nanoparticles are approved for clinical use (Table 2) or are currently investigated in clinical trials. Most clinically approved nanomedicines consist of liposomes as drug carriers including Doxil, DaunoXome, and the more recent VYXEOS (Table 2). Other types of nanoplatforms that are approved for clinical use involve chemotherapeutics bound to proteins and drugloaded polymers such as Eligard, which has been used to treat prostate cancer since 2002. Although most approved nanomedicine rely on passive targeting, targeted drug delivery gained attention to increase drug specificity. For instance, the antibody-drug conjugate Polivy has recently been approved to treat relapsed diffuse large B-cell lymphoma. Nevertheless, new nanoparticle platforms and approaches are emerging rapidly. Promising results have been obtained with new strategies such as small interfering RNA (siRNA) delivery to silence genes, targeted photoimmunotherapy (PIT), and synthetic dendritic cells to induce immune responses [6-8]. 
Table 2. Examples of clinically approved nanomedicine

\begin{tabular}{|c|c|c|c|c|c|}
\hline $\begin{array}{l}\text { Year of } \\
\text { approval }\end{array}$ & Name & $\begin{array}{l}\text { Nanotechnology } \\
\text { platform }\end{array}$ & $\begin{array}{l}\text { Therapeutic } \\
\text { agent }\end{array}$ & Indication & Refs \\
\hline $\begin{array}{l}1995 \text { (FDA) } \\
1996 \text { (EMA) }\end{array}$ & Doxil/Caelyx & Liposome (PEGylated) & Doxorubicin & $\begin{array}{l}\text { HIV-associated Kaposi sarcoma, } \\
\text { ovarian cancer, } \\
\text { metastatic breast cancer, multiple } \\
\text { myeloma }\end{array}$ & [9] \\
\hline 1996 (FDA) & DaunoXome & $\begin{array}{l}\text { Liposome (non- } \\
\text { PEGylated }\end{array}$ & Daunorubicin & HIV-associated Kaposi sarcoma & {$[10]$} \\
\hline 2002 (FDA) & Eligard & $\begin{array}{l}\text { poly }(D, L \text {-Lactide-co- } \\
\text { glycolide) }(P L G H)\end{array}$ & $\begin{array}{l}\text { Leuprorelin } \\
\text { acetate }\end{array}$ & Prostate cancer & [11] \\
\hline $\begin{array}{l}2005 \text { (FDA) } \\
2008 \text { (EMA) }\end{array}$ & Abraxane & $\begin{array}{l}\text { Nanoparticle albumin } \\
\text { bound }\end{array}$ & Paclitaxel & $\begin{array}{l}\text { Advanced non-small-cell lung cancer, } \\
\text { metastatic breast cancer, metastatic } \\
\text { pancreatic cancer }\end{array}$ & [12] \\
\hline 2006 (FDA) & Oncaspar & $\begin{array}{l}\text { polyethyleneglycol } \\
\text { (PEG) protein } \\
\text { conjugate }\end{array}$ & $L$-asparaginase & Leukemia & [13] \\
\hline 2009 (EMA) & MEPACT & $\begin{array}{l}\text { Liposome (non- } \\
\text { PEGylated) }\end{array}$ & Mifamurtide & Osteosarcoma & [14] \\
\hline 2015 (FDA) & $\begin{array}{l}\text { MM-398/ } \\
\text { Onivyde }\end{array}$ & Liposome (PEGylated) & Irinotecan & Metastatic pancreatic cancer & [15] \\
\hline 2017 (FDA) & VYXEOS & Liposome & $\begin{array}{l}\text { Daunorubicin } \\
\text { and cytarabine }\end{array}$ & Acute myeloid leukemia & {$[16]$} \\
\hline 2019 (FDA) & $\begin{array}{l}\text { Polivy } \\
\text { (polatuzumab } \\
\text { vedotin-piiq) }\end{array}$ & $\begin{array}{l}\text { Antibody-drug } \\
\text { conjugate }\end{array}$ & $\begin{array}{l}\text { Monomethyl } \\
\text { auristatin E }\end{array}$ & $\begin{array}{l}\text { Relapsed diffuse large B-cell } \\
\text { lymphoma }\end{array}$ & [17] \\
\hline
\end{tabular}

FDA: Food and Drug Administration; EMA: European Medicines Agency

Nanomedicine might seem the most appropriate approach in the fight against cancer, and could possibly even lead to the cure in the future. Yet, many challenges lie ahead, ranging from difficulties in design to limitations in clinical translation. And although promising, only a limited number of nanotechnologybased therapies has so far reached the clinic and still many nanomedicines fail to show benefits compared to conventional treatments $[18,19]$. Often this is due to lack of thorough experimental approaches that should evaluate the effects of nanomedicine on basic biological processes in cells and tissues. This review highlights recent advances that exploit unique promising features of polymeric nanoparticles and elaborates on remaining challenges (Figure 1).

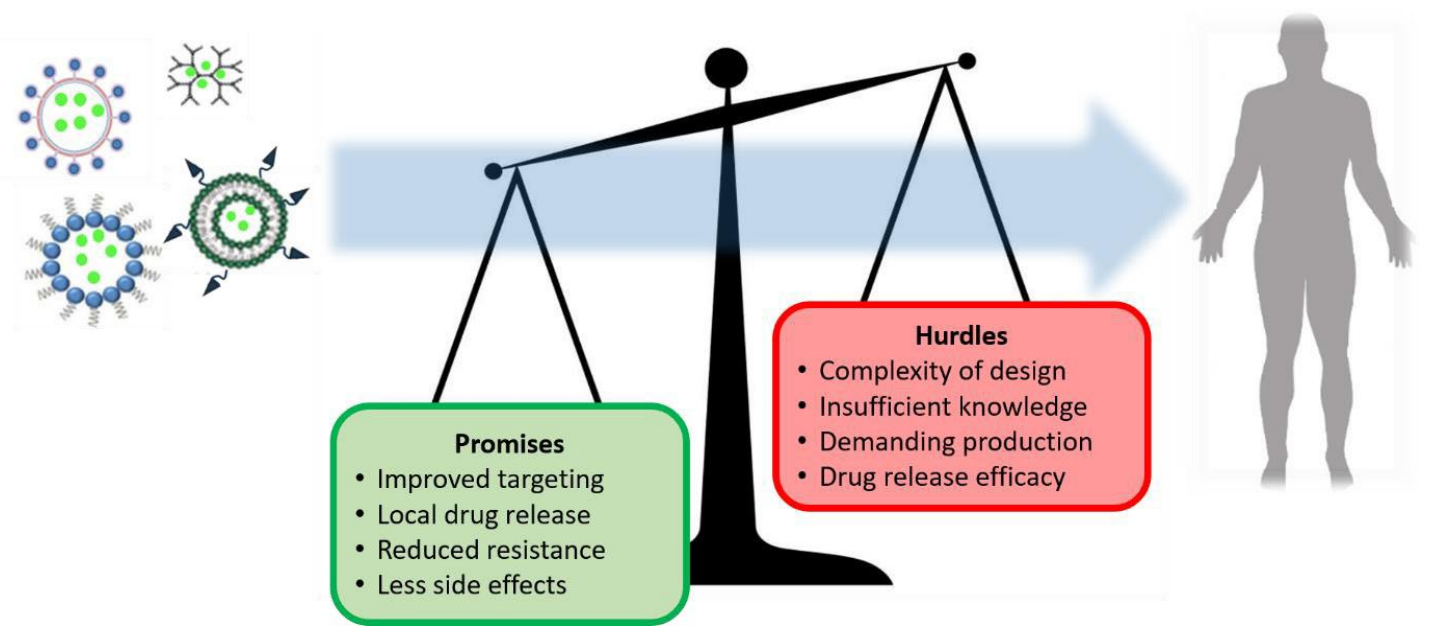

Figure 1. Schematic summary of promising aspects and remaining challenges of nanomedicine in cancer therapy

\section{Nanoparticle uptake and drug delivery}

Liposomes were the first nanoparticles that were approved for therapeutic use in the clinic. They have been used to increase the circulation time and specific uptake of the cytostatic drug doxorubicin [20, 21]. Free doxorubicin is an effective chemotherapeutic for several cancer types. However, the severe side effects due 
to cardiotoxicity limits the administered dose, resulting in low therapeutic efficacy [22]. Incorporation of doxorubicin in liposomes enhances uptake in tumor cells and results in a more gradual drug release, thereby preventing cardiotoxic effects [11,23-25]. Nevertheless, liposomal doxorubicin has failed to increase overall survival [26-28], which is likely attributed to the inefficient cargo release due to lack of triggered release after entering tumor tissue. This emphasizes the need for controlled release systems such as biodegradable drug carriers or modifications that allow drug release upon triggers from the tumor environment.

Nanoparticles have also attracted widespread interest as delivery vehicles for nucleic acids. siRNA is widely used to silence gene expression, but therapeutic application of free siRNA is limited due to its low stability in vivo [29]. Cationic polymers such as lipoplexes and polyplexes are commonly used as delivery vehicles, and are taken up by endocytotic pathways [30]. Encapsulation of siRNA in these nanoparticles increases stability, permeability, and cellular uptake [29, 31,32]. Therefore, many studies aimed to investigate the effects and mechanisms of siRNA nanoparticle delivery. Internalization of siRNA can occur through different mechanisms that are dependent on many properties of the polymeric vehicle, such as charge, size and conjugation of cell penetrating peptides, antibodies, or aptamers [33].

Knowledge on the mechanism of nanoparticle uptake is critical to aid development of siRNA release systems. The majority of nanoparticles are taken up by endocytosis due to their ability to interact with the cell membrane through properties such as receptor targeting ligands or a positive charge. The rapid acidification of endolysosomes causes siRNA degradation, and thus necessitates early endosomal release of siRNA. This has led to the development of nanoparticles functionalized with amines that are protonated at acidic pH, causing osmotic swelling and endosomal lysis [33]. This is also known as the proton sponge effect and although widely accepted, a small endosomal size and low membrane leakiness may be pivotal for sufficient build-up of osmotic pressure to allow proton sponge-based rupture [34]. Based on the comparison of four distinct cell types, endosomal rupture occurred less frequently in cells with smaller endosomes. Moreover, exposure to high polyplex concentrations increased endosomal leakiness, preventing endosomal rupture. In addition, Rejman et al. [35] reported caveolae-mediated endocytosis of polyplexes that may not involve endosomal acidification, indicating other release mechanisms than the proton sponge effect. Caveolae-mediated endocytosis was shown to be the main route for larger particles ( $>200 \mathrm{~nm})$, whereas smaller particles were exclusively internalized by clathrin-mediated endocytosis [35]. Alternative to the proton sponge effect, endosomal siRNA escape has been proposed to occur through pore formation by using endosomolytic peptides. Although studies report increased siRNA endosomal escape after incorporation of amines or endosomolytic peptides [36,37], internalization pathways and siRNA release mechanisms have been poorly addressed.

Live cell imaging has confirmed the association between cytosolic siRNA release and knockdown of targeted genes [38]. However, the release efficiency of nucleic acids from endosomes is considerably low with only a limited number of particles that release siRNA [30]. In addition, both lipoplexes and polyplexes do not rupture completely. Lipoplexes exhibited a more gradual release of siRNA in the cytosol, which could indicate endosomal release through pore formation. Nevertheless, siRNA nanoparticles were able to silence cancer associated genes in several tumor mouse models, leading to a significant decrease in tumor growth [39, 40]. siRNA treatment has also particularly attracted interest to treat triple-negative breast cancer (TNBC) that lack molecular features. Conventional drugs are ineffective because of the inability to target and the highly aggressive nature of TNBC. siRNA nanoparticles were able to silence different oncogenic genes in TNBC mice models [41].

Since escape from the endo-lysosomal system is a rate-limiting step for efficient drug delivery, modification of nanoparticles to bypass this system has gained interest $[42,43]$. As described above, nanoparticle size has important implications for uptake mechanisms and could be used to direct uptake to caveolae-mediated endocytosis and thereby prevent cargo degradation in lysosomes. Hence, it could improve nanoparticle cargo release $[35,42]$. Other strategies to mediate uptake by the caveolae pathway consist of modifying surface characteristics and shape [42]. Xin. et al. [44] developed nanoparticles that were able to bypass the endolysomal pathway by coating rod-like pure drug nanoparticles (PNPs) with hyaluronic acid (HA). These HA- 
PNPs were loaded with caspase 3 to assess protein delivery. The nanoparticles were able to enter cancer cells through binding to the CD44 receptor and uptake by the caveolar pathway. Levels of functional caspase- 3 increased by 7 -fold in human epithelial colorectal adenocarcinoma cell line, indicating efficient release. HA coating increased tumor accumulation in vivo, but the caveolae-mediated endocytosis was attributed to the rod-like shape $[44,45]$.

\section{Overcoming drug resistance}

A major problem in conventional cancer therapies is the development of drug resistance. Nanoparticles can prevent drug resistance by delivery of multiple drugs or siRNAs. Delivery of different siRNAs has potential to silence multiple genes simultaneously, including drug resistance genes. Nanoparticles that targeted the epidermal growth factor receptor (EGFR) on cancer cells, were successfully used for co-delivery of doxorubicin and siRNA to silence the drug resistance gene Bcl-2. After co-delivery, lung tumors in mice were more than twice smaller than those treated with doxorubicin or siRNA alone, indicating more effective suppression [6].

Multidrug resistance (MDR) is often caused by increased drug efflux or reduced uptake as a consequence of overexpressed transmembrane transporters. Aptamers, small single stranded DNA sequences, have shown potential to circumvent this resistance mechanism in cancer cells [46]. Liu et al. [47] developed a self-assembling DNA based nanostructure in which an aptamer was used to target nucleolin, a protein that is often upregulated in cancer cells. This was effectively used to deliver and retain doxorubicin in drug-resistant MCF-7 breast cancer cells. In vivo results demonstrated a tumor inhibition by $88 \%$ compared to an inhibition rate of $22 \%$ after administration of free doxorubicin [48]. The increased efficacy of nanomedicine in MDR is suggested to be a consequence of its different uptake mechanism. Unlike free drugs, targeted nanoparticles are taken up by endocytosis, which improves cellular uptake and circumvents drug efflux transporters.

Besides acquired MDR through increased drug efflux, intrinsic mechanisms including altered apoptosis signalling, play a major role in MDR [49]. Mitochondria have long been hypothesised to be involved in intrinsic MDR because of their role in energy metabolism and apoptosis. Studies have confirmed that an increase in oxidative phosphorylation (OXPHOS) in mitochondria is an important driver for chemoresistance [50-52]. This has stimulated the development of mitochondrial targeting nanoparticles to promote cell apoptosis by selective drug accumulation. Jiang et al. [53] generated paclitaxel containing liposomes that could successfully target cancer cells by the incorporation of 2, 3-dimethylmaleic anhydride (DMA), an amide bond that is cleaved in the acidic tumor microenvironment. Cleavage of DMA reverses nanoparticle charge, which promotes cellular uptake. Mitochondrial uptake was facilitated by the incorporation of the mitochondrial targeting peptide lysine-leucine-alanine and subsequently caused apoptosis in MDR lung cancer cells and in MDR lung cancer cell xenografted mice.

A recent study also demonstrated improved therapeutic outcomes in MDR tumors after mitochondrial drug accumulation [54]. Dendrigraft poly- $L$-lysine (DGL) nanoparticles, devised as carriers for doxorubicin, contained aptamers to target nucleolin on tumor cells and cytochrome $\mathrm{c}$ in mitochondria. Another aptamer allowed drug release that was triggered by the high concentrations of ATP in mitochondria. Mitochondrial drug release induced apoptosis in vitro and in vivo, indicating successful circumvention of acquired and intrinsic MDR by DGL nanoparticles.

Another mechanism of MDR involves the regulation of ceramide content in the cell membrane [55]. Ceramides are membrane lipids and regulate several cellular signalling pathways, including cell differentiation, proliferation, and programmed cell death. Overexpression of the enzyme glucosylceramide synthase, responsible for the conversion of the proapoptotic mediator ceramide to a nonfunctional glycosylceramide, is associated with MDR [55]. Co-administration of ceramide with the chemotherapeutic drug paclitaxel restored apoptotic signalling in MDR human ovarian cancer cells [56]. Moreover, inhibition of glucosylceramide synthase by the drug tamoxifen augmented the cytotoxic effects of paclitaxel in MDR human ovarian adenocarcinoma cells (by 10-fold) and non-MDR ovarian cancer cells (by more than 3-fold) [57]. This finding was confirmed by the increased anti-tumor efficiency after the addition of tamoxifen to paclitaxel-loaded nanoparticles in a xenograft mouse model. So in addition to specific targeting to circumvent MDR, the ability 
to co-deliver compounds to restore altered signalling pathways is a promising feature of nanomedicine to overcome MDR [57]. Knowledge about the particular MDR mechanism in patients is therefore crucial to decide the best nanoparticle design for the treatment of chemo-resistant tumors.

\section{Strategies of controlled drug release}

When nanoparticles are taken up in the cell, they need to release the drug to evoke therapeutic effects. The design of effective release strategies is one of the main challenges encountered in nanomedicine. Endocytosis is the major route for nanoparticle internalization but cargo release is known to be a rate-limiting step and necessitated the need for controlled drug release [58]. Specific characteristics of the tumor microenvironment and endosomes, including the acidic $\mathrm{pH}$ and metabolic products, have been exploited to trigger drug release. Furthermore, nanoparticles that release their cargo upon external stimuli such as exposure to ultrasound, light, temperature, or magnetic fields have shown promising as controlled drug release systems [59-64].

\section{Sustained drug release}

Biodegradable polymers allow sustained drug release. Both natural and synthetic polymers are used as drug release systems, but synthetic polymers offer the advantage of a more gradual and controlled release. Polymer degradation causes the release of encapsulated drugs and can be controlled by altering the composition and molecular weight $[65,66]$. Polylactides (PLA) and poly ( $D, L$-lactide-co-glycolide) (PLGA) are the most extensively studied non-toxic polymers for controlled drug release [67]. These particles can be easily altered to control the rate of hydrolysis and thereby allow sustained drug release over a period of days to several weeks. Although highly suitable for drug release, PLA and PLGA nanoparticles are not biocompatible with all drugs [68]. Other biodegradable materials are therefore investigated to cover the wide range of available drugs. Polymers such as modified poly(glycerol-adipate) (PGAS), PEGylated poly( $\varepsilon$-caprolactone) (PEGPCL) or calcium-based biomaterials are promising materials for drug release systems because of their high tunability and low toxicity [69-72].

\section{Stimuli-responsive drug delivery}

For localized drug release in cancer cells, several nanoparticles have been designed to release their cargo upon tumor specific stimuli [61]. Cyclodextrin-based nanoplatforms have various biomedical applications for cancer treatment and theranostics because different functionalities can be incorporated to make them responsive to stimuli, such as $\mathrm{pH}$, temperature, redox, enzymes, light and magnetic fields [73]. In addition, polymers and liposomes can be functionalized with groups that alter particle charge after environmental change in $\mathrm{pH}$, causing disruption and subsequent drug release. A recent study demonstrated selective release in tumor cells, triggered by the acidic lysosomal $\mathrm{pH}$ and high levels of glutathione in cervical cancer cells [74]. Rhodamine B-modified chitosan was used as a model for macromolecular drugs and aminated fluorescein served as a model for small molecule drugs. Both substances were incorporated into biodegradable organosilica-based core-shell structured nanoparticles, and were only released after uptake in tumor cells. This indicates its potential as a controlled dual drug delivery system. However, in vivo studies are needed to confirm the efficacy and specificity of this drug release system.

Polymers and liposomes have also been used as building blocks for nanoparticles that release their cargo upon exposure to endogenous enzymes [75]. Furthermore, the use of glucose oxidase has attracted increasing interest due to its potential to enhance conventional nanomedicine strategies [75]. Glucose oxidase efficiently catalyzes the oxidization of glucose into hydrogen peroxide and gluconic acid, which can trigger pH-responsive drug release by increasing the acidity of the tumor microenvironment. Moreover, hydrogen peroxide can be converted into toxic hydroxyl radicals that may enhance the toxicity of PIT [76]. Because particles need different characteristics to accumulate in the tissue and internalize in cells, several studies explored hierarchical targeting approaches that exploit changeable particle sizes, switchable charge and activatable surface ligand [77, 78]. These nanoparticles are generally not activated during blood circulation to facilitate passive tumor accumulation, and are then reactivated by external or internal stimuli to stimulate cellular uptake. 
As examples of exogenous stimuli applied to control drug release, photosensitive and magnetic release systems are widely investigated because of their convenience to trigger localized release by exposure of light or a magnetic field $[62,79,80]$. Near-infrared (NIR) light provides advantages for its higher tissue penetration as compared to light in the visible spectrum. NIR light was able to trigger release of doxorubicin from poly (ether amine) nanoparticles that contained the dye cyanine [59].31.8\% of doxorubicin was released after the first four minutes of NIR light exposure and release increased to almost $60 \%$ after three cycles of repeated irradiation with one exposure of four minutes every hour, whereas almost no release was seen when NIR light exposure was stopped. In mice with hepatocellular carcinoma, excitation of cyanine was followed by effective tumor cytotoxicity, which was attributed to both photothermal effects and the thermoresponsive release of doxorubicin.

Recent advances show the synergistic effect of combining chemotherapy, photodynamic therapy (PDT) and hyperthermia therapy [81]. Superior cytotoxic effects in vitro were observed after triggering magnetic liposomes that contained the photosensitizer meta-tetra(hydroxyphenyl)chlorin (mTHPC) and the drug doxorubicin [60]. Exposure of a magnetic field induced magnetic hyperthermia that also triggered heat sensitive liposomal doxorubicin release. Additional light exposure to excite m-THPC, almost completely eliminated tumor cells after $12 \mathrm{~h}$ of combined therapy. One study that also shows the potential in vivo was executed by Li et al. [82], who designed a human epidermal growth factor receptor 2-targeting liposome with a modified photosensitizer (ICG-ODA). After 5 min of NIR light exposure, ICG-ODA could generate sufficient reactive oxygen species for PDT and triggered release of incorporated doxorubicin in vitro. A maximum of $80 \%$ of doxorubicin was released in $4 \mathrm{~h}$ after irradiation, but was highly dependent on the ratio of ICG-ODA and total lipid. Mice treated with this dual therapy had an enhanced anti-tumor effect when compared to PDT or doxorubicin treatment alone, indicating both effective PDT and drug release in vivo. Also ultrasound irradiation has shown to enhance the uptake and anti-tumor activity of human serum albumin-bound paclitaxel loaded with the photosensitizer sinoporphyrin sodium [83]. Combining these three modalities was associated with near total arrest of tumor growth inhibition in a tumor mouse model as compared to controls without ultrasound irradiation.

\section{Nanomedicine in immunotherapy}

One of the main therapeutic approaches in cancer is to provoke anti-tumor responses by stimulating the immune response. Clinical success of immunotherapy has been achieved for many cancer types, but the highly heterogeneous responses have attracted interest to image immune cell behavior [84]. An important application of nanoparticles is therefore to visualize distribution and monitor efficacy of cell based immunotherapies, for example by loading immune cells with imaging particles such as iron oxide or perfluorocarbon agents for magnetic resonance imaging (MRI) [85]. Imaging particles have provided valuable insights about the localization and interaction of tumor infiltrating immune cells, leading to improvements in immunotherapy. Although different therapeutic approaches have led to great progress, current immunotherapies are limited by their high costs, heterogeneous responses and lack of control. These limitations have recently stimulated the design of nano-sized artificial immune cells, which will be discussed in the next paragraph. Another promising approach for controlled modulation of the immune system is local delivery of immunotherapeutic agents or immune cells by biomaterial scaffold-based drug delivery systems, such as implanted scaffold and injected hydrogels [86]. Other applications of therapeutic nanoparticles in immunotherapy are reviewed by Jia et al. [87].

Recent advances in the design of artificial antigen-presenting cells (aAPCs) are promising to stimulate anti-tumor responses and can overcome many of the limitations of current immunotherapies $[8,88]$. Vaccination of natural dendritic cells (DCs) is commonly used to stimulate the immune response but lead to different responses between patients. These heterogenous responses are likely the result of differences in DC signalling and molecular expression. Synthetic variants on the other hand, have opportunities for full control over $\mathrm{T}$ cell activation and overcome the need to produce a customized vaccine for each patient. Synthetic DCs could therefore reduce costs by providing off-the-shell therapy in the future. 
The design of aAPCs is inspired by natural dendritic cells and can stimulate T cells by incorporation of the required signals: signal 1 to activate T cell receptors, signal 2 consisting of co-stimulatory molecules and signal 3 provided by cytokines. The potential of aAPCs is shown by recent progress of nanosized filaments to stimulate $\mathrm{T}$ cells. These nanosized filaments, consisting of a poly (isocyano peptide) co-polymer, were functionalized to attach signalling molecules that elicited strong sustained T cell activation in vitro [8, 89] (Figure 2). Future studies will most likely be directed to unravel the mechanisms of T cell activation by aAPCs, so design can be optimized to obtain full control over $\mathrm{T}$ cell response.

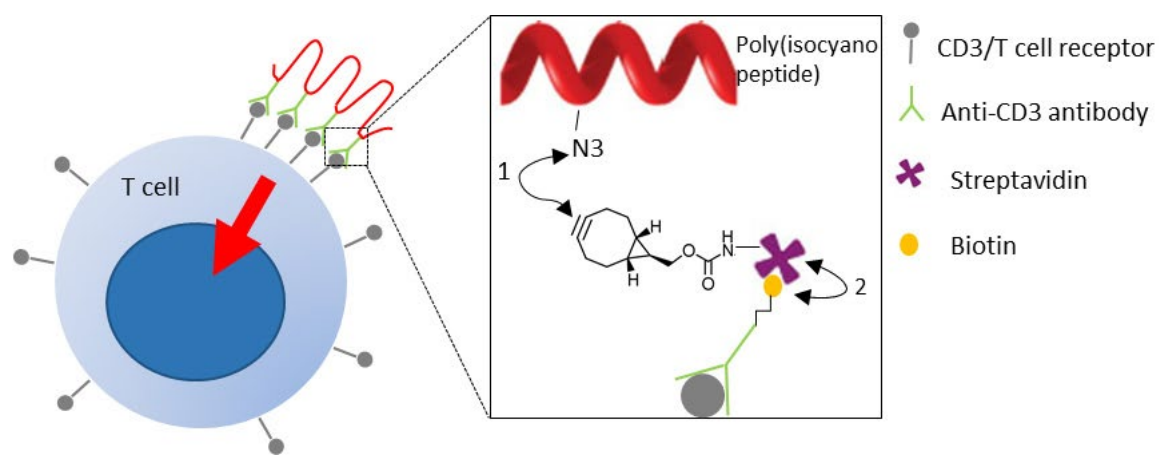

Figure 2. Artificial antigen presenting cells to activate T cells. Poly (isocyano peptide) is conjugated to an anti-CD3 antibody that activates T cell signalling upon binding T cell receptors. (1) Azide groups of poly (isocyano peptide) were coupled to bicyclononynefunctionalized streptavidin through a strain-promoted azide-alkyne click reaction (SPAAC); (2) streptavidin binds to biotinylated anti-CD3 antibodies

Another strategy in immunotherapy exploits light to kill cancer cells. Phototherapy has long been known for its cytotoxic effects, but only in the last decade the combination of light with chemicals are used to treat cancer [90]. In conventional PDT, a photosensitizing agent becomes activated on excitation by light. As it falls back to the ground state, the activated photosensitizer transfers energy to oxygen molecules. This leads to the generation of singlet and reactive oxygen species that mediate tissue damage [90].

Because PDT also evokes damage in healthy tissue, a targeted photosensitizer has been developed. In this approach, known as PIT, a monoclonal antibody is conjugated to a photosensitizer that is excited by NIR light (Figure 3). Mitsunaga et al. [7] conjugated the photosensitizer IR-700 to the HER1 receptor targeting antibody panitumumab. Upon excitation, panitumumab-IR-700 induced targeted specific cell death. In vivo injection of panitumumab-IR-700 and subsequent irradiation by NIR light, led to a significant decrease in volume of HER1 positive tumors [7].

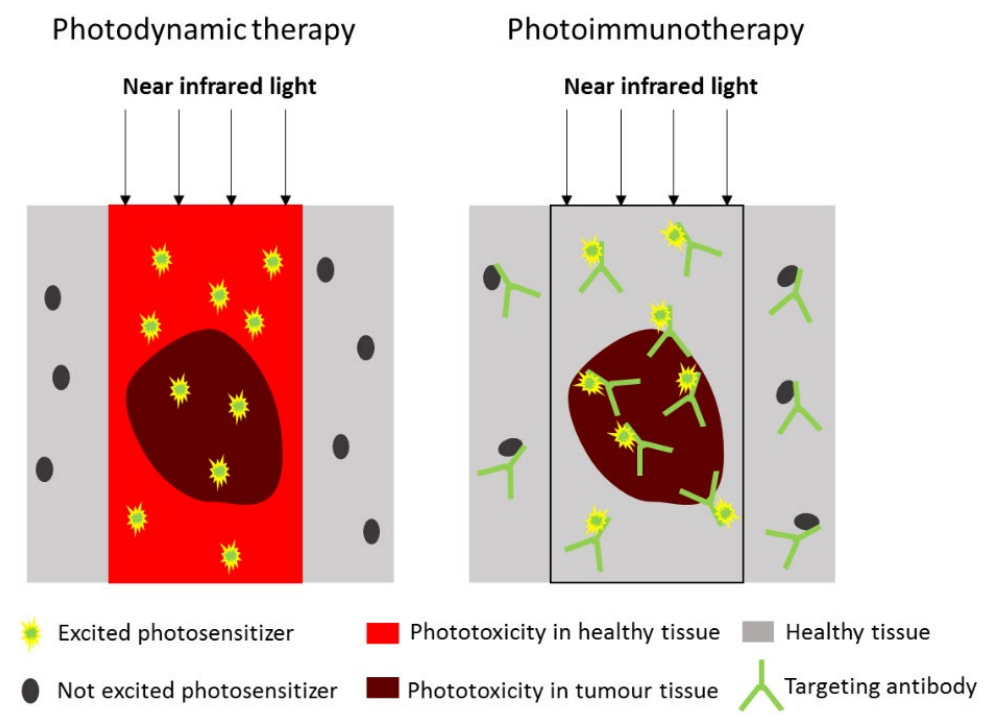

Figure 3. Difference between PDT and PIT. In PDT, a photosensitizer induces widespread tissue damage upon excitation by NIR light (left). In PIT, the photosensitizer is attached to a monoclonal antibody that specifically targets molecules expressed on cancer cells. Upon excitation, the photosensitizer only induces cell death when bound to the cell membrane (right) 
Since the first success of PIT, also other targets have been used, including EGFR, mesothelin (hYP218) and prostate specific membrane antigen (PSMA) [91-94]. These studies also showed positive results with decreased tumor size and prolonged survival in vivo. A phase 2 clinical trial on the EGFR targeting cetuximabIR-700 conjugate (RM-1929) is currently ongoing in the USA to determine efficacy in head and neck cancer [95].

A recent study indicates that the effect of PIT largely depends on induction of anti-tumor immunity. PIT rapidly caused damage that led to disposal of antigens and immunogenic signals, which was followed by maturation of dendritic cells [96]. This order of events is known to be associated with an anti-tumor response. However, no direct evidence was provided for the role of the host immune system in the effectivity of PIT. Studies in immunodeficient mice might elucidate the exact contribution of the host immune system in the effect of PIT [97]. Since immune escape mechanisms are an important hallmark of cancer, the application of nanoparticles to stimulate host immunity to cancer may lead to high therapeutic response.

\section{Remaining challenges}

Debates about the hype or hope of nanomedicine are ongoing and represent the controversy in the field. Nanomedicine might have suffered from high expectations, causing skepticism after lagging results. Yet, we cannot deny the first promising results. The ability to improve pharmacokinetics and modify properties has potential to increase drug efficacy, while limiting side effects in patients. Some nanomedicines have already shown clinical success, but the application in daily practice is still in its infancy. Despite the emergence of a tremendous number of designs for nanoparticle-based drug delivery, only a few are approved for clinical use. It is essential to understand the underlying reasons for this poor clinical translation to address remaining challenges.

\section{Search for the optimal design}

High failure rates of nanomedicine can be partly attributed to unforeseen interactions in the body that cause limited specific uptake and high toxicity. It is therefore of utmost importance to understand the effects of different physicochemical properties on the behavior in biological environments. These insights are also necessary to improve design. However, the combination of design considerations to generate targeted, effective and also safe nanomedicine, is complex due to the interdependence of different properties and main functionalities. PEGylation might for example increase drug circulation time, but inhibits cellular uptake and endosomal escape $[29,98]$. To overcome this phenomenon, known as the 'PEG dilemma', various nanocarriers have been developed that cleave PEG upon stimuli $[99,100]$. The PEG dilemma emphasizes the need to overcome possible detrimental effects of beneficial properties. Small differences in properties such as size, charge and PEG density could have large effects on biodistribution and uptake [101-103]. Therefore, properties of nanoparticles should be accurately characterized and described. Two techniques that are commonly used to measure size and charge of nanoparticles are dynamic light scattering and zeta potential but concerns have been raised regarding their use and data interpretation [104]. Variation in data quality between studies makes it difficult to interpret and reproduce results. Therefore, it is important to standardize protocols for nanoparticle characterization. This could substantially increase the understanding of how nanoparticle characteristics influence interactions in the body, which is essential knowledge to improve design.

Another difficulty in design, arises from the demand to cross multiple different barriers in the body. Nanoparticles need to have different functionalities in each different environment. Clearance of nanoparticles should first of all be avoided to reach the tumor vasculature, whereas leakage to tumor tissue and subsequent accumulation depend on the EPR effect. Free drugs should then reach the target, either by release after cellular uptake or by diffusion through the tumor microenvironment. The final drug concentration at the target site is highly dependent on the efficiency of cellular uptake and controlled drug release. Knowledge about the nanoparticle-cell membrane interface and subsequent internalization pathways is inevitable to predict potential nanomedicine efficacy and toxicity, and to promote clinical translation. 
All the desirable effects should be considered to generate effective nanomedicine but may be challenging to combine. Nanoparticle design will be facilitated by knowledge from other disciplines such as cell biology and cancer pathophysiology. The importance of this biological knowledge for nanomedicine design has already been illustrated by considering the particle surface to prevent opsonization or increase uptake, the size to allow the EPR effect, controlled drug release by triggers in the tumor microenvironment, and the influence of membrane composition on cellular uptake. Although these insights can improve basic design considerations in general, there is no 'one size fits all answer'. Because of the highly heterogeneous nature of cancer, personalized strategies will become important to achieve optimal therapeutic efficacy in each individual.

\section{Optimizing the EPR effect}

The accumulation of nanoparticles in tumor tissue is attributed to the EPR effect, but disappointing clinical results have questioned the significance of this effect. The EPR effect is often exaggerated in animal models because of induced tumor growth and substantial differences from human cancer [105] (Figure 4). The extent of the EPR effect in human, is in fact highly heterogeneous between patients and cancer types [106]. For instance, hepatocellular carcinoma generally has a high EPR effect, whereas this effect is limited in pancreatic and prostate cancer because of low vascular density that leads to hypoxic areas. Furthermore, a high tumor tissue density leads to compression of blood vessels, which increases the interstitial fluid pressure which acts as a counter pressure in the extravasation of nanomedicine.

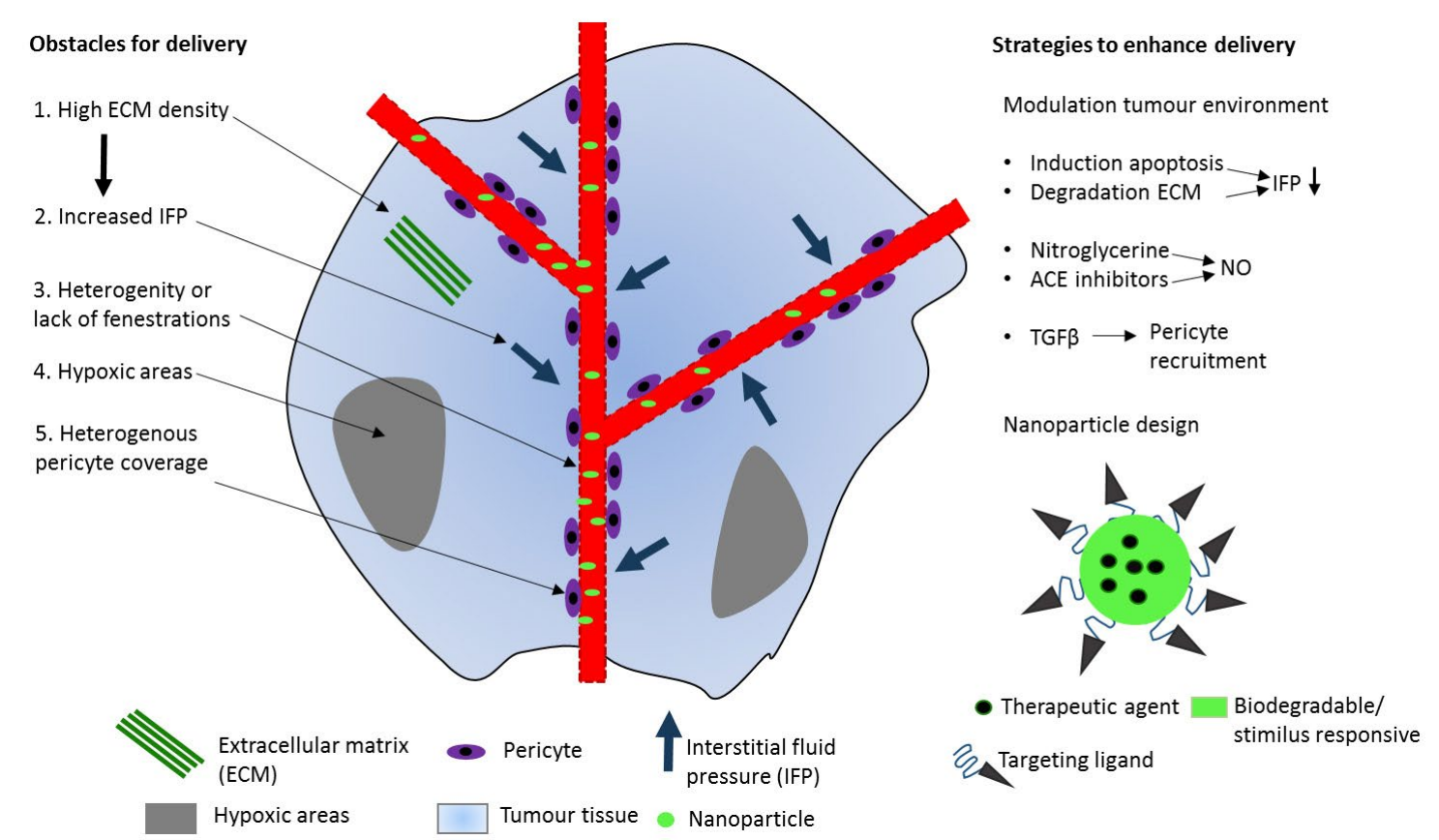

Figure 4. Obstacles and strategies in drug delivery to tumor tissue. Five factors in the human tumor microenvironment that limit nanomedicine delivery to tumor tissue (left). These five features are distinct from murine tumors and might therefore explain the lower drug delivery efficiency in humans when compared to animal models. Strategies to enhance the EPR effect by modulating the tumor microenvironment (upper right). Drug delivery can also be improved by modifying nanoparticles to increase cellular uptake and drug release at the target (lower right). ACE: angiotensin-converting enzyme; NO: nitric oxide; TGF: transforming growth factor

Modulating the tumor microenvironment to enhance the EPR effect is promising (Figure 4) [107]. Induction of tumor cell apoptosis or extracellular matrix (ECM) degradation in high density tumor tissues could lead to a decreased interstitial fluid pressure by vessel decompression. The cytotoxic drug paclitaxel has shown to increase the EPR effect in pre-clinical and clinical studies [108-110]. Induction of tumor cell apoptosis led to blood vessel decompression in vivo and also demonstrated decreased interstitial fluid pressure in breast cancer patients. The EPR effect can also be enhanced by increasing the vessel permeability. Agents that generate NO mediate vascular permeability and release local NO in hypoxic areas, resulting in and enhanced drug delivery by 2 to 3 -fold [111]. Another strategy to increase vascular permeability is by 
targeting the pericyte coverage of tumor endothelium. TGF- $\beta$ inhibitors increase vessel leakiness by inhibition of pericyte recruitment to tumor vessels and improved both delivery of $100 \mathrm{~nm}$ nanoparticles and anti-tumor activity [112].

The improved delivery by enhancing the EPR effect suggests that nanomotors might also have potential to facilitate accumulation in tumor tissue. However, for selective accumulation it is essential that these nanomotors move towards a specific tumor substrate. Non-specific uptake in healthy tissues is a common problem of nanoparticles in general, and often evokes toxicity. This is especially a concern in healthy tissues that also possess the EPR effect such as liver and spleen. To limit toxicity, nanoparticles and their biodegradation products should consist of biocompatible non-toxic materials. Toxicological assessments and research on their fate is important for safety analysis and can elucidate mechanisms of toxicity.

\section{The paradox of active targeting}

After nanomedicine accumulates in tumor tissue, cellular uptake is needed to evoke therapeutic effects. Active targeting can increase selective cellular uptake and is especially beneficial when drugs have intracellular targets. Moreover, active targeting has potential to circumvent drug resistance mechanisms [47]. However, its added value has been subjected to debate. Functionalized liposomes have been criticized for their increased clearance by the immune system [113]. When compared to passive targeting, the shorter circulation time of targeted nanomedicine might lead to less drug accumulation in tumor tissue. To clarify possible drawbacks of active targeting, studies should not only compare the efficacy of novel targeting approaches to free drugs, but also to their non-targeted counterpart. And although results are usually not generalized for different particles and cell types, it is important to realize that study outcomes might differ because of distinct uptake mechanisms. The type of endocytotic pathway is largely dependent on particle characteristics, targeting moiety, and cell type [114]. So extensive studies should be carried out for every new particle design or when the same nanoparticles are evaluated in a different cancer type.

Another issue that seems contradictory, involves the disappointing results of using targeting moieties with high avidity. High avidity nanoparticles are generally believed to be advantageous but are limited by their low tumor penetration [115]. Small antibody fragments and peptide ligands with weak affinity show higher tumor penetration, which is associated with higher efficacy [113]. Taken together, these results emphasize the need to consider effects on clearance and tumor penetration in the design of active targeting strategies.

The efficiency of active targeting not only depends on nanoparticle characteristics, but also largely depends on the target. The target should be highly abundant in tumor tissue, while expression in healthy tissue should be low. Molecular expression on cancer cells is highly heterogeneous within and between patients. It is therefore important to characterize cancer cells and identify appropriate targets. Nanomedicine can then be adjusted to each patient and different targets can be used to address heterogeneity within one patient. Moreover, nanotheranostic capsules could help to identify reasonable molecular targets and establish optimal dose. In addition to molecular targets, other interindividual distinctions in the tumor microenvironment, such as in vascularisation and ECM density, should also be considered to reinforce therapy (Figure 3). However, this requires careful tumor characterization, which may be challenging when biopsies are not possible.

\section{Increasing effective drug release}

The ineffective drug release is one of the major limitations of current nanomedicine and gave rise to controlled drug release systems. Various stimuli responsive nanoparticles have shown to increase selective drug release at the target [116]. Although it is useful to know more about the possibilities and working mechanisms of triggered drug release, many of these systems are not suitable for clinical use. For instance, heat triggered release might not be possible in human because of safety issues from increased temperatures. Moreover, the use of magnetic fields to trigger drug release might not be feasible in long-term treatment because of the inconvenience and high costs that are associated with the high frequency of patient exposure. Although frequency of nanomedicine administration might be kept to a minimum by prolonged and sustained drug release. Drug release by internal triggers from the tumor microenvironment might be more appropriate due to its use of natural triggers. However, this would require very sensitive systems that only release drugs on 
tumor specific stimuli. Co-triggered systems, such as $\mathrm{pH} /$ glutathione sensitive nanoparticles could therefore increase specificity of release. Nevertheless, heterogeneous responses can be expected because of interpatient differences in tumor environment.

\section{Increasing clinical translation}

Although more clinical success in cancer nanomedicine is known (reviewed in [117]), the amount of nanomedicine that reaches the clinic is low. Most progress can likely be achieved from taking into account clinical translation in fundamental research. Novel nanobased designs are increasingly emerging at a high pace but often neglect potential clinical application. For this purpose, it is important to design non-toxic adjustable nanoparticles with limited production requirements. These modular delivery platforms are essential to reduce costs and to enable bulk production. Another important aspect to decrease the translation gap, is the use of pre-clinical models that adequately resemble the human condition. Advanced in vitro models, such as tumors-on-a-chip, are promising to evaluate nanomedicine and could significantly facilitate clinical translation $[118,119]$.

\section{Conclusions}

Taken together, research has indicated the difficulty to achieve targeted, effective, safe, and at the same time, easy to produce nanomedicine. Many challenges lie ahead, but the promising results indicate great potential in personalized cancer treatment. Before nanomedicine can actually lead to a revolution in cancer therapy, a multidisciplinary approach should be adopted by combining knowledge from different disciplines including cell biology, tumor pathophysiology and chemistry. Besides in cancer therapy, nanomedicine tools have been developed for the treatment of several autoimmune and inflammatory diseases (reviewed in [120-122]). The lessons learned for the development of nanomedicine-based delivery strategies of immunomodulatory agents and anti-inflammatory molecules will certainly benefit the field of cancer and vice versa.

While this review mostly focused on polymeric nanoparticles, it is worth mentioning the development of prodrug based nanomedicines, which can controllably release drugs at specific sites under specific stimulation (see [123] for in-depth review). The so-called prodrug (i.e. the drug conjugated to a pre carrier) is an inactive compound that can be locally and controllably cleaved or degraded into the real bioactive drug directly in vivo [123]. Numerous prodrug-based nanomedicines with different chemical modifications have been developed that are responsive to both endogenous stimuli $[124,125]$ and exogenous environmental stimuli [126], thus expanding the repertoire of nanomedicines available to treat cancer.

Progress in nanomedicine would require a better understanding of nanoparticle behavior in biological environments, a more careful characterization to facilitate reproducibility, and increased focus on clinical application. Expanding our knowledge will facilitate improvements in design, ultimately leading to more efficient and less toxic drugs. Nanomedicine might then live up to its promise and substantially improve patient outcomes in the future.

\section{Abbreviations}

aAPCs: artificial antigen-presenting cells

EGFR: epidermal growth factor receptor

EMA: European Medicines Agency

EPR: enhanced permeability and retention

FDA: Food and Drug Administration;

HA: hyaluronic acid

MDR: multidrug resistance

NIR: near-infrared

NO: nitric oxide 
PDT: photodynamic therapy

PEG: polyethyleneglycol

PIT: photoimmunotherapy

PLGA: poly $(D, L$-lactide-co-glycolide)

siRNA: small interfering RNA

TNBC: triple-negative breast cancer

\section{Declarations}

\section{Author contributions}

All authors contributed to manuscript writing and revision, read and approved the submitted version.

\section{Conflicts of interest}

The authors declare that they have no conflicts of interest.

Ethical approval

Not applicable.

\section{Consent to participate}

Not applicable.

\section{Consent to publication}

Not applicable.

Availability of data and materials

Not applicable.

\section{Funding}

Not applicable.

\section{Copyright}

(c) The Author(s) 2021.

\section{References}

1. Gerlinger M, Swanton C. How darwinian models inform therapeutic failure initiated by clonal heterogeneity in cancer medicine. Br J Cancer. 2010;103:1139-43.

2. Gottesman MM. Mechanisms of cancer drug resistance. Annu Rev Med. 2002;53:615-27.

3. Lungu II, Grumezescu AM, Volceanov A, Andronescu E. Nanobiomaterials used in cancer therapy: an upto-date overview. molecules 2019;24:3547.

4. Stylianopoulos T, Jain RK. Design considerations for nanotherapeutics in oncology. Nanomedicine. 2015;11:1893-907.

5. Piktel E, Niemirowicz K, Watek M, Wollny T, Deptula P, Bucki R. Recent insights in nanotechnology-based drugs and formulations designed for effective anti-cancer therapy. J Nanobiotechnology. 2016;14:39.

6. Zhang X, Wang Q, Qin L, Fu H, Fang Y, Han B, et al. EGF-modified mPEG-PLGA-PLL nanoparticle for delivering doxorubicin combined with Bcl-2 siRNA as a potential treatment strategy for lung cancer. Drug Deliv. 2016;23:2936-45.

7. Mitsunaga M, Ogawa M, Kosaka N, Rosenblum LT, Choyke PL, Kobayashi H. Cancer cell-selective in vivo near infrared photoimmunotherapy targeting specific membrane molecules. Nat Med. 2012;17:1685-91. 
8. Hammink R, Mandal S, Eggermont LJ, Nooteboom M, Willems PH, Tel J, et al. Controlling T-cell activation with synthetic dendritic cells using the multivalency effect. ACS Omega. 2017;2:937-45.

9. Barenholz YC. Doxil囚—the first FDA-approved nano-drug: lessons learned. J Control Release. 2012;160:117-34.

10. Rosenthal E, Poizot-Martin I, Saint-Marc T, Spano JP, Cacoub P, Group DS. Phase IV study of liposomal daunorubicin (DaunoXome) in AIDS-related Kaposi sarcoma. Am J Clin Oncol. 2002;25:57-9.

11. Braeckman J, Michielsen D. Efficacy and tolerability of 1- and 3-month leuprorelin acetate depot formulations (Eligard $($ /Depo-Eligard $®$ ) for advanced prostate cancer in daily practice: a Belgian prospective non-interventional study. Arch Med Sci. 2014;10:477-83.

12. Saif MW. US Food and Drug Administration approves paclitaxel protein-bound particles (Abraxane $®$ ) in combination with gemcitabine as first-line treatment of patients with metastatic pancreatic cancer. JOP. 2013;14:686-8.

13. Dinndorf PA, Gootenberg J, Cohen MH, Keegan P, Pazdur R. FDA drug approval summary: pegaspargase (Oncaspar $\circledast$ ) for the first-line treatment of children with acute lymphoblastic leukemia (ALL). Oncologist. 2007;12:991-8.

14. Meyers PA. Muramyl tripeptide (mifamurtide) for the treatment of osteosarcoma. Expert Rev Anticancer Ther. 2009;9:1035-49.

15. Zhang H. Onivyde for the therapy of multiple solid tumors. Onco Targets Ther. 2016;9:3001.

16. Nikanjam M, Capparelli EV, Lancet JE, Louie A, Schiller G. Persistent cytarabine and daunorubicin exposure after administration of novel liposomal formulation CPX-351: population pharmacokinetic assessment. Cancer Chemother Pharmacol. 2018;81:171-8.

17. Deeks ED. Polatuzumab vedotin: ffirst global approval. Drugs. 2019;79:1467-75.

18. Bobo D, Robinson KJ, Islam J, Thurecht KJ, Corrie SR. Nanoparticle-based medicines: a review of FDAapproved materials and clinical trials to date. Pharm Res. 2016;33:2373-87.

19. Caster JM, Patel AN, Zhang T, Wang A. Investigational nanomedicines in 2016: a review of nanotherapeutics currently undergoing clinical trials. Wiley Interdiscip Rev Nanomed Nanobiotechnol. 2017;9.

20. Gabizon A, Shiota R, Papahadjopoulos D. Pharmacokinetics and tissue distribution of doxorubicin encapsulated in stable liposomes with long circulation times. J Natl Cancer Inst. 1989;81:1484-8.

21. Theodoulou M, Batist G, Campos S, Winer E, Welles L, Hudis C. Phase I study of nonpegylated liposomal doxorubicin plus trastuzumab in patients with HER2-positive breast cancer. Clin Breast Cancer. 2009;9:101-7.

22. Swain SM, Whaley FS, Ewer MS. Congestive heart failure in patients treated with doxorubicin: a retrospective analysis of three trials. Cancer. 2003;97:2869-79.

23. Uziely B, Jeffers S, Isacson R, Kutsch K, Wei-Tsao D, Yehoshua Z, et al. Liposomal doxorubicin: antitumor activity and unique toxicities during two complementary phase I studies. J Clin Oncol. 1995;13:1777-85.

24. Muggia FM, Hainsworth JD, Jeffers S, Miller P, Groshen S, Tan M, et al. Phase II study of liposomal doxorubicin in refractory ovarian cancer: antitumor activity and toxicity modification by liposomal encapsulation. J Clin Oncol. 1997;15:987-93.

25. Safra T, Muggia F, Jeffers S, Tsao-Wei D, Groshen S, Lyass O, et al. Pegylated liposomal doxorubicin (doxil): reduced clinical cardiotoxicity in patients reaching or exceeding cumulative doses of $500 \mathrm{mg} / \mathrm{m}^{2}$. Ann Oncol. 2000;11:1029-33.

26. Wagner U, Marth C, Largillier R, Kaern J, Brown C, Heywood M, et al. Final overall survival results of phase III GCIG CALYPSO trial of pegylated liposomal doxorubicin and carboplatin vs paclitaxel and carboplatin in platinum-sensitive ovarian cancer patients. Br J Cancer. 2012;107:588-91. 
27. Halm U, Etzrodt G, Schiefke I, Schmidt F, Witzigmann H, Mössner J, et al. A phase II study of pegylated liposomal doxorubicin for treatment of advanced hepatocellular carcinoma. Ann of Oncol. 2000;11: 113-4.

28. O'brien M, Wigler N, Inbar M, Rosso R, Grischke E, Santoro A, et al. Reduced cardiotoxicity and comparable efficacy in a phase III trial of pegylated liposomal doxorubicin $\mathrm{HCl}$ (CAELYX ${ }^{\mathrm{TM}} /$ Doxil@) versus conventional doxorubicin for first-line treatment of metastatic breast cancer. Ann Oncol. 2004;15:440-9.

29. Mishra S, Webster P, Davis ME. PEGylation significantly affects cellular uptake and intracellular trafficking of non-viral gene delivery particles. Eur J Cell Biol. 2004;83:97-111.

30. Rehman Zu, Hoekstra D, Zuhorn IS. Mechanism of polyplex-and lipoplex-mediated delivery of nucleic acids: real-time visualization of transient membrane destabilization without endosomal lysis. ACS Nano. 2013;7:3767-77.

31. Urban-Klein B, Werth S, Abuharbeid S, Czubayko F, Aigner A. RNAi-mediated gene-targeting through systemic application of polyethylenimine (PEI)-complexed siRNA in vivo. Gene Ther. 2005;12:461-6.

32. Ragelle H, Danhier F, Préat V, Langer R, Anderson DG. Nanoparticle-based drug delivery systems: a commercial and regulatory outlook as the field matures. Expert Opin Drug Deliv. 2017;14:851-64.

33. Arnold AE, Czupiel P, Shoichet M. Engineered polymeric nanoparticles to guide the cellular internalization and trafficking of small interfering ribonucleic acids. J Control Release. 2017;259:3-15.

34. Vermeulen LMP, Brans T, Samal SK, Dubruel P, Demeester J, De Smedt SC, et al. Endosomal size and membrane leakiness influence proton sponge-based rupture of endosomal vesicles. ACS Nano. 2018;12:2332-45.

35. Rejman J, Conese M, Hoekstra D. Gene transfer by means of lipo-and polyplexes: role of clathrin and caveolae-mediated endocytosis. J Liposome Res. 2006;16:237-47.

36. Agarwal S, Zhang Y, Maji S, Greiner A. PDMAEMA based gene delivery materials. Materials Today. 2012;15:388-93.

37. Cheng $Q$, Du L, Meng L, Han S, Wei T, Wang X, et al. The promising nanocarrier for doxorubicin and siRNA co-delivery by PDMAEMA-based amphiphilic nanomicelles. ACS Appl Mater Interfaces. 2016;8:4347-56.

38. Wittrup A, Ai A, Liu X, Hamar P, Trifonova R, Charisse K, et al. Visualizing lipid-formulated siRNA release from endosomes and target gene knockdown. Nat Biotechnol. 2015;33:870-6.

39. Li Z, Chen G, Ding L, Wang Y, Zhu C, Wang K, et al. Increased survival by pulmonary treatment of established lung metastases with dual STAT3/CXCR4 inhibition by siRNA nanoemulsions. Mol Ther. 2019;27:2100-10.

40. Babu A, Muralidharan R, Amreddy N, Mehta M, Munshi A, Ramesh R. Nanoparticles for siRNA-based gene silencing in tumor therapy. IEEE Trans Nanobioscience. 2016;15:849-63.

41. Parvani JG, Jackson MW. Silencing the roadblocks to effective triple-negative breast cancer treatments by siRNA nanoparticles. Endocr Relat Cancer. 2017;24:R81-97.

42. Foroozandeh P, Aziz AA. Insight into Cellular Uptake and Intracellular Trafficking of Nanoparticles. Nanoscale research letters. 2018;13:339.

43. Blanco E, Shen H, Ferrari M. Principles of nanoparticle design for overcoming biological barriers to drug delivery. Nat Biotechnol. 2015;33:941-51.

44. Xin X, Teng C, Du X, Lv Y, Xiao Q, Wu Y, et al. Drug-delivering-drug platform-mediated potent protein therapeutics via a non-endo-lysosomal route. Theranostics. 2018;8:3474-89.

45. Zhang L, Yang X, Lv Y, Xin X, Qin C, Han X, et al. Cytosolic co-delivery of miRNA-34a and docetaxel with core-shell nanocarriers via caveolae-mediated pathway for the treatment of metastatic breast cancer. Sci Rep. 2017;7:46186.

46. Bar-Zeev M, Livney YD, Assaraf YG. Targeted nanomedicine for cancer therapeutics: towards precision medicine overcoming drug resistance. Drug Resist Updat. 2017;31:15-30. 
47. Liu J, Wei T, Zhao J, Huang Y, Deng H, Kumar A, et al. Multifunctional aptamer-based nanoparticles for targeted drug delivery to circumvent cancer resistance. Biomaterials. 2016;91:44-56.

48. Yu S, Bi X, Yang L, Wu S, Yu Y, Jiang B, et al. Co-delivery of paclitaxel and PLK1-targeted siRNA using aptamer-functionalized cationic liposome for synergistic anti-breast cancer effects in vivo. J Biomed Nanotechnol. 2019;15:1135-48.

49. Guerra F, Arbini AA, Moro L. Mitochondria and cancer chemoresistance. Biochim Biophys Acta Bioenerg. 2017;1858:686-99.

50. Guaragnella N, Giannattasio S, Moro L. Mitochondrial dysfunction in cancer chemoresistance. Biochem Pharmacol. 2014;92:62-72.

51. Farge T, Saland E, De Toni F, Aroua N, Hosseini M, Perry R, et al. Chemotherapy-resistant human acute myeloid leukemia cells are not enriched for leukemic stem cells but require oxidative metabolism. Cancer Discov. 2017;7:716-35.

52. De Luca A, Fiorillo M, Peiris-Pagès M, Ozsvari B, Smith DL, Sanchez-Alvarez R, et al. Mitochondrial biogenesis is required for the anchorage-independent survival and propagation of stem-like cancer cells. Oncotarget. 2015;6:14777-95.

53. Jiang L, Li L, He X, Yi Q, He B, Cao J, et al. Overcoming drug-resistant lung cancer by paclitaxel loaded dualfunctional liposomes with mitochondria targeting and pH-response. Biomaterials. 2015;52:126-39.

54. Chen H, Tian J, Liu D, He W, Guo Z. Dual aptamer modified dendrigraft poly-L-lysine nanoparticles for overcoming multi-drug resistance through mitochondrial targeting. J Mater Chem B. 2017;5:972-9.

55. Itoh M, Kitano T, Watanabe M, Kondo T, Yabu T, Taguchi Y, et al. Possible role of ceramide as an indicator of chemoresistance: decrease of the ceramide content via activation of glucosylceramide synthase and sphingomyelin synthase in chemoresistant leukemia. Clin Cancer Res. 2003;9:415-23.

56. van Vlerken LE, Duan Z, Seiden MV, Amiji MM. Modulation of intracellular ceramide using polymeric nanoparticles to overcome multidrug resistance in cancer. Cancer Res. 2007;67:4843-50.

57. Devalapally H, Duan Z, Seiden MV, Amiji MM. Modulation of drug resistance in ovarian adenocarcinoma by enhancing intracellular ceramide using tamoxifen-loaded biodegradable polymeric nanoparticles. Clin Cancer Res. 2008;14:3193-203.

58. Shete HK, Prabhu RH, Patravale VB. Endosomal escape: a bottleneck in intracellular delivery. J Nanosci Nanotechnol. 2014;14:460-74.

59. Kedmi R, Veiga N, Ramishetti S, Goldsmith M, Rosenblum D, Dammes N, et al. A modular platform for targeted RNAi therapeutics. Nat Nanotechnol. 2018;13:214-9.

60. Shah SA, Khan MA, Arshad M, Awan S, Hashmi M, Ahmad N. Doxorubicin-loaded photosensitive magnetic liposomes for multi-modal cancer therapy. Colloids Surf B Biointerfaces. 2016;148:157-64.

61. Sun T, Zhang YS, Pang B, Hyun DC, Yang M, Xia Y. Engineered nanoparticles for drug delivery in cancer therapy. Angew Chem Int Ed Engl. 2014;53:12320-64.

62. He H, Zhou J, Liu Y, Liu S, Xie Z, Yu M, et al. Near-infrared-light-induced morphology transition of poly (ether amine) nanoparticles for supersensitive drug release. ACS Appl Mater Interfaces. 2018;10:7413-21.

63. Nittayacharn P, Yuan HX, Hernandez C, Bielecki P, Zhou H, Exner AA. Enhancing tumor drug distribution with ultrasound-triggered nanobubbles. J Pharm Sci. 2019;108:3091-8.

64. Zhou C, Xie X, Yang H, Zhang S, Li Y, Kuang C, et al. Novel class of ultrasound-triggerable drug delivery systems for the improved treatment of tumors. Mol Pharm. 2019;16:2956-65.

65. Göpferich A. Mechanisms of polymer degradation and erosion. Biomaterials. 1996;17:103-14.

66. Lin SY, Chen KS, Teng HH, Li MJ. In vitro degradation and dissolution behaviours of microspheres prepared by three low molecular weight polyesters. J Microencapsul. 2000;17:577-86.

67. Panyam J, Labhasetwar V. Biodegradable nanoparticles for drug and gene delivery to cells and tissue. Adv Drug Deliv Rev. 2003;55:329-47. 
68. Lucke A, Kiermaier J, Gopferich A. Peptide acylation by poly(alpha-hydroxy esters). Pharm Res. 2002;19:175-81.

69. Weiss VM, Lucas H, Mueller T, Chytil P, Etrych T, Naolou T, et al. Intended and unintended targeting of polymeric nanocarriers: the case of modified poly (glycerol adipate) nanoparticles. Macromol Biosci. 2018;18:1700240.

70. Grossen P, Witzigmann D, Sieber S, Huwyler J. PEG-PCL-based nanomedicines: a biodegradable drug delivery system and its application. J Control Release. 2017;260:46-60.

71. Swainson SME, Taresco V, Pearce AK, Clapp LH, Ager B, McAllister M, et al. Exploring the enzymatic degradation of poly(glycerol adipate). Eur J Pharm Biopharm. 2019;142:377-86.

72. Qi C, Lin J, Fu LH, Huang P. Calcium-based biomaterials for diagnosis, treatment, and theranostics. Chem Soc Rev. 2018;47:357-403.

73. Yao X, Mu J, Zeng L, Lin J, Nie Z, Jiang X, et al. Stimuli-responsive cyclodextrin-based nanoplatforms for cancer treatment and theranostics. Mater Horiz. 2019;6:846-70.

74. Li JL. Dual drug delivery system based on biodegradable organosilica core-shell architectures. ACS Appl Mater Interfaces. 2018;10:5287-95.

75. Mu J, Lin J, Huang P, Chen X. Development of endogenous enzyme-responsive nanomaterials for theranostics. Chem Soc Rev. 2018;47:5554-73.

76. Fu LH, Qi C, Lin J, Huang P. Catalytic chemistry of glucose oxidase in cancer diagnosis and treatment. Chem Soc Rev. 2018;47:6454-72.

77. Wang S, Huang P, Chen X. Stimuli-responsive programmed specific targeting in nanomedicine. ACS Nano. 2016;10:2991-4.

78. Wang S, Huang P, Chen X. Hierarchical targeting strategy for enhanced tumor tissue accumulation/ retention and cellular internalization. Adv Mater. 2016;28:7340-64.

79. Ju Y, Tong S, Hou Y. Stimuli-regulated cancer theranostics based on magnetic nanoparticles. In: Clin Appl Magnetic Nanoparticles. 1st ed. New York: CRC Press; 2018.p.22.

80. Bansal A, Zhang Y. Photocontrolled nanoparticle delivery systems for biomedical applications. Acc Chem Res. 2014;47:3052-60.

81. Fan W, Yung B, Huang P, Chen X. Nanotechnology for multimodal synergistic cancer therapy. Chem Rev. 2017;117:13566-638.

82. Li Q, Li W, Di H, Luo L, Zhu C, Yang J, et al. A photosensitive liposome with NIR light triggered doxorubicin release as a combined photodynamic-chemo therapy system. J Control Release. 2018;277:114-25.

83. Zhang Y, Wan Y, Chen Y, Blum NT, Lin J, Huang P. Ultrasound-enhanced chemo-photodynamic combination therapy by using albumin "nanoglue"-based nanotheranostics. ACS Nano. 2020;14:5560-9.

84. Zanganeh S, Spitler R, Hutter G, Ho JQ, Pauliah M, Mahmoudi M. Tumor-associated macrophages, nanomedicine and imaging: the axis of success in the future of cancer immunotherapy. Immunotherapy. 2017;9:819-35.

85. Zeelen C, Paus C, Draper D, Heskamp S, Signore A, Galli F, et al. In vivo imaging of tumor-infiltrating immune cells: implications for cancer immunotherapy. Q J Nucl Med Mol Imaging. 2018;62:56-77.

86. Yang C, Blum NT, Lin J, Qu J, Huang P. Biomaterial scaffold-based local drug delivery systems for cancer immunotherapy. Science Bulletin. 2020;65:1489-504.

87. Jia Y, Omri A, Krishnan L, McCluskie MJ. Potential applications of nanoparticles in cancer immunotherapy. Hum Vaccin Immunother. 2017;13:63-74.

88. Schluck M, Hammink R, Figdor CG, Verdoes M, Weiden J. Biomaterial-based activation and expansion of tumor-specific T cells. Front Immunol. 2019;10:931.

89. Mandal S, Eksteen-Akeroyd ZH, Jacobs MJ, Hammink R, Koepf M, Lambeck AJ, et al. Therapeutic nanoworms: towards novel synthetic dendritic cells for immunotherapy. Chem Sci. 2013;4:4168-74. 
90. Dolmans DE, Fukumura D, Jain RK. Photodynamic therapy for cancer. Nat Rev Cancer. 2003;3:380-7.

91. Nakajima Takahito T. Improving the efficacy of Photoimmunotherapy (PIT) using a cocktail of antibody conjugates in a multiple antigen tumor model. Theranostics. 2013;3:357-65.

92. Nagaya T, Nakamura Y, Sato K, Zhang YF, Ni M, Choyke PL, et al. Near infrared photoimmunotherapy with an anti-mesothelin antibody. Oncotarget. 2016;7:23361-9.

93. Watanabe R, Hanaoka H, Sato K, Nagaya T, Harada T, Mitsunaga M, et al. Photoimmunotherapy targeting prostate-specific membrane antigen: are antibody fragments as effective as antibodies? J Nucl Med. 2015;56:140-4.

94. Nishimura T, Mitsunaga M, Ito K, Kobayashi H, Saruta M. Cancer neovasculature-targeted near-infrared photoimmunotherapy (NIR-PIT) for gastric cancer: different mechanisms of phototoxicity compared to cell membrane-targeted NIR-PIT. Gastric Cancer. 2020;23:82-94.

95. Cognetti D, Curry J, Gillenwater A, William W, Kochuparambil S, McDonald D, et al. A phase 2a, multicenter, open-label study of RM-1929 photoimmunotherapy in patients with recurrent head and neck cancer. Int J Radiat Oncol. 2018;100:1368.

96. Ogawa M, Tomita Y, Nakamura Y, Lee MJ, Lee S, Tomita S, et al. Immunogenic cancer cell death selectively induced by near infrared photoimmunotherapy initiates host tumor immunity. Oncotarget. 2017;8:10425-36.

97. Nakajima K, Takakura H, Shimizu Y, Ogawa M. Changes in plasma membrane damage inducing cell death after treatment with near-infrared photoimmunotherapy. Cancer Sci. 2018;109:2889-96.

98. Xia Y, Tian J, ChenX. Effect of surface properties on liposomal siRNA delivery. Biomaterials. 2016;79:56-68.

99. Hatakeyama H, Akita H, Harashima H. A multifunctional envelope type nano device (MEND) for gene delivery to tumours based on the EPR effect: a strategy for overcoming the PEG dilemma. Adv Drug Deliv Rev. 2011;63:152-60.

100. Fang Y, Xue J, Gao S, Lu A, Yang D, Jiang H, et al. Cleavable PEGylation: a strategy for overcoming the "PEG dilemma" in efficient drug delivery. Drug Deliv. 2017;24:22-32.

101. Shi J, Kantoff PW, Wooster R, Farokhzad OC. Cancer nanomedicine: progress, challenges and opportunities. Nat Rev Cancer. 2017;17:20-37.

102. Walkey CD, Olsen JB, Guo H, Emili A, Chan WC. Nanoparticle size and surface chemistry determine serum protein adsorption and macrophage uptake. J Am Chem Soc. 2012;134:2139-47.

103. Badkas A, Frank E, Zhou Z, Jafari M, Chandra H, Sriram V, et al. Modulation of in vitro phagocytic uptake and immunogenicity potential of modified Herceptin ${ }^{\circledR}$-conjugated PLGA-PEG nanoparticles for drug delivery. Colloids Surf B Biointerfaces. 2018;162:271-8.

104. Bhattacharjee Sourav S. DLS and zeta potential-what they are and what they are not? J Control Release. 2016;235:337-51.

105. Danhier F. To exploit the tumor microenvironment: since the EPR effect fails in the clinic, what is the future of nanomedicine? J Control Release. 2016;244:108-21.

106. Maeda Hiroshi H. Toward a full understanding of the EPR effect in primary and metastatic tumors as well as issues related to its heterogeneity. Adv Drug Deliv Rev. 2015;91:3-6.

107. Zhang B, Hu Y. Modulating the tumor microenvironment to enhance tumor nanomedicine delivery. Front Pharmacol. 2017;8:952.

108. Griffon-Etienne G, Boucher Y, Brekken C, Suit HD, Jain RK. Taxane-induced apoptosis decompresses blood vessels and lowers interstitial fluid pressure in solid tumors: clinical implications. Cancer Res. 1999;59:3776-82.

109. Danhier F, Danhier P, Magotteaux N, De Preter G, Ucakar B, Karroum O, et al. Electron paramagnetic resonance highlights that the oxygen effect contributes to the radiosensitizing effect of paclitaxel. PLoS One. 2012;7:e40772. 
110. Taghian AG, Abi-Raad R, Assaad SI, Casty A, Ancukiewicz M, Yeh E, et al. Paclitaxel decreases the interstitial fluid pressure and improves oxygenation in breast cancers in patients treated with neoadjuvant chemotherapy: clinical implications. J Clin Oncol. 2005;23:1951-61.

111. Seki T, Fang J, Maeda H. Enhanced delivery of macromolecular antitumor drugs to tumors by nitroglycerin application. Cancer Sci. 2009;100:2426-30.

112. Cabral H, Matsumoto Y, Mizuno K, Chen Q Murakami M, Kimura M, et al. Accumulation of sub-100 nm polymeric micelles in poorly permeable tumours depends on size. Nat Nanotechnol. 2011;6:815-23.

113. Noble GT, Stefanick JF, Ashley JD, Kiziltepe T, Bilgicer B. Ligand-targeted liposome design: challenges and fundamental considerations. Trends Biotechnol. 2014;32:32-45.

114. Voltan AR, Alarcon KM, Fusco-Almeida AM, Soares CP, Mendes-Giannini MJ, Chorilli M. Highlights in Endocytosis of Nanostructured Systems. Curr Med Chem. 2017;24:1909-29.

115. Bazak R, Houri M, El Achy S, Kamel S, Refaat T. Cancer active targeting by nanoparticles: a comprehensive review of literature. J Cancer Res Clin Oncol. 2015;141:769-84.

116. Ramasamy T, Ruttala HB, Gupta B, Poudel BK, Choi HG, Yong CS, et al. Smart chemistry-based nanosized drug delivery systems for systemic applications: a comprehensive review. J Control Release. 2017;258:226-53.

117. Tran S, DeGiovanni PJ, Piel B, Rai P. Cancer nanomedicine: a review of recent success in drug delivery. Clin Transl Med. 2017;6:44.

118. Zhang YS, Zhang YN, Zhang W. Cancer-on-a-chip systems at the frontier of nanomedicine. Drug Discov Today. 2017;22:1392-9.

119. Bhise NS, Ribas J, Manoharan V, Zhang YS, Polini A, Massa S, et al. Organ-on-a-chip platforms for studying drug delivery systems. J Control Release. 2014;190:82-93.

120. Gharagozloo M, Majewski S, Foldvari M. Therapeutic applications of nanomedicine in autoimmune diseases: from immunosuppression to tolerance induction. Nanomedicine. 2015;11:1003-18.

121. Feng X, Liu J, Xu W, Li G, Ding J. Tackling autoimmunity with nanomedicines. Nanomedicine. 2020; 15:1585-97.

122. Brusini R, Varna M, Couvreur P. Advanced nanomedicines for the treatment of inflammatory diseases. Adv Drug Deliv Rev. 2020;157:161-78.

123. Xie A, Hanif S, Ouyang J, Tang Z, Kong N, Kim NY, et al. Stimuli-responsive prodrug-based cancer nanomedicine. EBioMedicine. 2020;56:102821.

124. Ling X, Tu J, Wang J, Shajii A, Kong N, Feng C, et al. Glutathione-responsive prodrug nanoparticles for effective drug delivery and cancer therapy. ACS Nano. 2019;13:357-70.

125. Sun B, Luo C, Yu H, Zhang X, Chen Q, Yang W, et al. Disulfide bond-driven oxidation- and reductionresponsive prodrug nanoassemblies for cancer therapy. Nano Lett. 2018;18:3643-50.

126. Dai Y, Xiao H, Liu J, Yuan Q Ma Pa, Yang D, et al. In vivo multimodality imaging and cancer therapy by near-infrared light-triggered trans-platinum pro-drug-conjugated upconverison nanoparticles. J Am Chem Soc. 2013;135:18920-9. 